\title{
On a Class of Multiple Failure Mode Systems
}

\author{
Michael V. Boutsikas and Markos V. Koutras* \\ University of Piraeus, Greece
}

\begin{abstract}
The primary objective of this work is to introduce and perform a detailed study of a class of multistate reliability structures in which no ordering in the levels of components' performances is necessary. In particular, the present paper develops the basic theory (exact reliability formulae, reliability bounds, asymptotic results) that will make it feasible to investigate systems whose components are allowed to experience $m \geq 2$ kinds of failure (failure modes) and their breakdown is described by different families of cut sets in each mode. For illustration purposes, two classical (binary) systems are extended to analogous multiple failure mode structures and their reliability performance (bounds and asymptotic behaviour) is investigated by numerical experimentation.

Keywords and Phrases: Reliability bounds; negative association; coherent structures; $k$-out-of- $n$ systems; multiple failure mode systems; minimal cut sets.
\end{abstract}

1991 subject classifications: Primary 60K10, Secondary 60E15

\section{Introduction}

The vast majority of reliability analyses deals with binary systems, i.e., systems whose components can assume one of two states (operating or failed). However, in many practical settings, the systems' components can realize more than two modes of operation. For example, in fluid control networks, a defective valve could be either "stuck-open" or "stuck-closed"; in electronic/electrical engineering studies, a non-working relay circuit can be either "failed-open" or "failed-short (closed)"; in safety monitoring systems, a device's malfunction usually refers either to "failure to detect breakdown" or to "initiation of a false alarm". For systems experiencing two different kinds of failure, the most popular nomenclature in use seems to be three-state devices or dual failure mode (DFM) systems. Specific structures of this type have been studied among others by Barlow, Hunter and Proschan [4], Ben-Dov [5], Misra [19], Satoh, Sasaki, Yuge and Yanasi [23] and Koutras [16].

During the last three decades, significant contributions were made towards building up a theory of multistate systems consisting of multistate components. This direction of research was motivated by the observation that many real life systems are more realistically modelled by allowing their components to assume a (finite) number of states, say $0,1, \ldots, m$, each corresponding to a different level of operating efficiency. Key references in this field are Barlow and Wu [3], El-Neweihi, Proschan and Sethuraman [10], Ross [21], Griffith [14], Natvig [20] and Meng [18]. A basic ingredient in their analyses is that the operating modes of the components (and the system) should be ordinable, i.e., the $m+1$ states represent successive levels of performance ranging from perfect functioning down

\footnotetext{
${ }^{*}$ Research partially supported by the Greek General Secretariat for Research and Technology (GRST) under grant $70 / 3 / 2948$
} 
to complete failure. Apparently, this is not always true in real life; for example, none of the DFM systems described earlier can be modeled as a multistate system (for $m=2$ ) in the framework put forward so far.

In the present article, motivated by this last observation, we introduce a family of multistate systems which are not subject to the restriction of state ordering. The approach we take to achieve this goal is based on an appropriate extension of the definition of an ordinary binary reliability system through minimal cut sets. More specifically, let $I$ be the set of components of the system and label the working state of each component $i \in I$ by 0 and the different failure modes of it by $1,2, \ldots, m$. Next, assign to each failure mode $s=1,2, \ldots, m$, a family of minimal cut sets $\mathbf{C}_{s} \subset \mathcal{P}(I)$ characterized by the following property: if $C \in \mathbf{C}_{s}$ then the simultaneous trapping of all $i \in C$ to failure mode $s$ initiates system's breakdown. Apparently the components' set $I$ along with the families of minimal cut sets $\mathbf{C}_{1}, \mathbf{C}_{2}, \ldots, \mathbf{C}_{m}$ provides a well defined reliability structure. The name we shall be using for such structures is multiple failure mode (MFM) systems so as to distinguish them from the multistate systems mentioned earlier. The DFM systems studied by Barlow, Hunter and Proschan [4], Ben-Dov [5] etc. are special cases of $M F M$ systems with $m=2$. Moreover, for $m=1$, our definition reduces to the classical set theoretic definition of binary systems (through minimal cut sets); throughout this paper we shall also use for them the term single failure mode $(S F M)$ systems.

The primary objective of this work is to provide an exhaustive study of the class of $M F M$ systems including techniques for exact reliability evaluation, construction of high quality reliability bounds and investigation of the asymptotic behaviour of large systems.

We shall now summarize the contents of the present article. In Section 2 we present the formal definition of an MFM system and introduce the notation and terminology used through the paper. Section 3 discusses two different approaches for the exact reliability evaluation; the first makes use of appropriate binary functions whereas the second reduces the problem to the computation of the reliability of a $S F M$ system. In Section 4 we derive several lower and upper reliability bounds which are subsequently used to investigate (in Section 5) the asymptotic behaviour of large $M F M$ systems. Section 6 deals with two classes of $M F M$ systems which have been deduced by natural extensions to a multifailure environment of ideas involved in classical $S F M$ systems. Methods for evaluating the associated reliability bounds are discussed and extensive numerical calculations are carried out in order to illustrate the accuracy of the estimation achieved.

In closing we mention that all definitions and results presented in this article could be effortlessly modified so that a minimal path set (instead of minimal cut set) approach be taken. However, since most of the outcomes can be easily formulated and justified by employing duality arguments, we shall not pursue this matter further, leaving the details to the interested readers.

\section{Definitions and notations}

The structure of a $S F M$ system with a finite set of components $I$ can be uniquely described by the so-called structure function $\phi:\{0,1\}^{|I|} \rightarrow\{0,1\}$ which maps the set of components' states into the set of system's possible states (0:failed, 1:functioning). For coherent systems, where $\phi$ is coordinatewise nondecreasing, the structure function can be written in the form (see e.g. Barlow 
and Proschan [2])

$$
\phi(\mathbf{y})=\prod_{C \in \mathbf{C}}\left(1-\prod_{i \in C}\left(1-y_{i}\right)\right)
$$

where $\mathbf{y}=\left(y_{i}, i \in I\right) \in\{0,1\}^{|I|}$ represents the components' state, $\mathbf{C}=$ minimal $\left\{\left\{i \in I: y_{i}=\right.\right.$ $\left.0\}: \mathbf{y} \in \phi^{-1}(0)\right\}$, and minimal(A) stands for the set of all minimal sets of $\mathbf{A}$ with respect to the partial ordering $\subset$. The elements of the family $\mathbf{C}$ are known as the minimal cut sets of the system. Obviously, if the random variable $Y_{i} \in\{0,1\}$ represents the state of the $i$-th component, $i \in I$, and $\mathbf{Y}=\left(Y_{i}, i \in I\right)$, then system's reliability can be expressed as $P(\phi(\mathbf{Y})=1)=E(\phi(\mathbf{Y}))$.

In the present article we shall consider a generalization of the $S F M$ coherent systems by allowing the components to experience $m$ different kinds of failure. To start with, let $S \cup\{0\}, S=\{1,2, \ldots, m\}$ be the set of states of each component $i \in I$, with 0 representing the components's working state and $1, \ldots, m$ indicating several malfunction states (failure modes). At a specific instance $t$, each of the components can only be in one of the $m+1$ mutually exclusive and exhaustive states of $S \cup\{0\}$. The survival probability (at time $t$ ) of component $i \in I$ will be denoted by $p_{i}$, while the probability that component $i$ is in state $s \in S$ will be denoted by $q_{s i}, s=1,2, \ldots, m$. Note that time $t$ has been suppressed in the notation, since it will be kept fixed throughout our exposition.

As already announced in the introduction, our definition for multiple failure mode $(M F M)$ systems will be established by considering several families of cut sets, each family being associated with a different failure mode. To be more precise, we assume that we have at hand $m$ minimal subsets $\mathbf{C}_{1}, \mathbf{C}_{2}, \ldots, \mathbf{C}_{m}$ of $\mathcal{P}(I)$ such that the system fails if and only if there exists $s \in S$ and $C \in \mathbf{C}_{s}$ with all components in $C$ experiencing (at time $t$ ) failure in mode $s$. Such a failure will be labelled as system failure in mode $s$ while the elements of $\mathbf{C}_{s}$ will be called minimal cut sets of type $s$.

The state of the components of the system could be described by a family of integer valued random variables $V_{i}, i \in I$ defined on $\{0\} \cup S$ with their distributions given by $P\left(V_{i}=0\right)=$ $p_{i}, P\left(V_{i}=s\right)=q_{s i}, s \in S$. In the sequel it will be proved much more fruitful to describe the state of the components by a binary random matrix $\mathbf{X}=\left(X_{s i}\right)$ defined as follows

$$
X_{s i}=\left\{\begin{array}{l}
0, \text { if component } i \text { is in failure mode } s \\
1, \text { otherwise. }
\end{array}, i \in I, s \in S\right.
$$

For typographical convenience, in the statement of the general results of the next paragraphs, we shall be assuming that $I=\{1,2, \ldots, n\}$. The $m n$-dimensional random variables generated by the rows of matrix $\mathbf{X}$ will be denoted by $\mathbf{X}_{s .}, s \in S$ while the $n m$-dimensional random columns of $\mathbf{X}$ will be denoted by $\mathbf{X}_{\cdot i}^{\prime}, i \in I$. Because of the assumption that the states of $S \cup\{0\}$ are exhaustive and mutually exclusive, it is clear that the random variables $\mathbf{X}_{. i}^{\prime}, i \in I$ can only take on the values $\mathbf{1}^{\prime}, \mathbf{1}^{\prime}-\mathbf{e}_{1}^{\prime}, \ldots, \mathbf{1}^{\prime}-\mathbf{e}_{m}^{\prime}\left(\mathbf{e}_{s}^{\prime}, s \in S\right.$ are the unit column vectors of $\mathbb{R}^{n}$ and $\left.\mathbf{1}^{\prime}=\sum_{s=1}^{m} \mathbf{e}_{s}^{\prime}\right)$. Clearly, $\mathbf{1}^{\prime}-\mathbf{X}_{\cdot i}^{\prime}=\mathbf{e}_{s}^{\prime}$ if and only if component $i$ is experiencing a failure of type $s$ ( $i$ is in failure mode $s$ ) while $\mathbf{1}^{\prime}-\mathbf{X}_{\cdot i}^{\prime}=\mathbf{0}^{\prime}$ if and only if component $i$ is working. Therefore, for all $i \in I$,

$$
P\left(\mathbf{1}^{\prime}-\mathbf{X}_{\cdot i}^{\prime}=\mathbf{0}^{\prime}\right)=p_{i}=P\left(V_{i}=0\right), \quad P\left(\mathbf{1}^{\prime}-\mathbf{X}_{\cdot i}^{\prime}=\mathbf{e}_{s}^{\prime}\right)=q_{s i}=P\left(V_{i}=s\right), \quad s \in S .
$$

In the sequel we assume that the components of the system are working independently. As a consequence, the random vectors $\mathbf{X}_{\cdot i}^{\prime}, i=1,2, \ldots, n$ are statistically independent.

In closing this section let us introduce the notation $\phi_{s}(\cdot)$ for a $S F M$ structure function of the form (1) with $\mathbf{C}=\mathbf{C}_{s}, s \in S$, i.e.

$$
\phi_{s}(\mathbf{y})=\prod_{C \in \mathbf{C}_{s}}\left(1-\prod_{i \in C}\left(1-y_{i}\right)\right) .
$$


It is readily ascertainable that the system failure in mode $s$ is equivalent to $\phi_{s}\left(\mathbf{X}_{s}\right)=0$. Therefore, the probability that the system is not in failure mode $s$ equals $E\left(\phi_{s}\left(\mathbf{X}_{s}\right)\right)$ which is a function of $\mathbf{q}_{s .}=\left(q_{s 1}, q_{s 2}, \ldots, q_{s n}\right)$ and will be denoted by $R_{s}\left(\mathbf{q}_{s .}\right)$, i.e.,

$$
R_{s}\left(\mathbf{q}_{s .}\right)=R_{s}\left(q_{s 1}, q_{s 2}, \ldots, q_{s n}\right)=E\left(\phi_{s}\left(\mathbf{X}_{s}\right)\right) .
$$

Note that $R_{s}\left(\mathbf{q}_{s}\right), s \in S$ are actually reliabilities of $S F M$ systems with component failure probabilities $\mathbf{q}_{s .}=\left(q_{s 1}, q_{s 2}, \ldots, q_{s n}\right)$ and minimal cut sets $C \in \mathbf{C}_{s}$.

\section{Exact reliability evaluation}

By the definition of the multiple failure mode system introduced in the previous section, the system fails if and only if $\phi_{s}\left(\mathbf{X}_{s}\right)=0$ for at least one $s \in S$. Hence, the binary function $\phi_{M F M}:\{0,1\}^{m \times n} \rightarrow\{0,1\}$ defined by

$$
\phi_{M F M}(\mathbf{X})=\prod_{s=1}^{m} \phi_{s}\left(\mathbf{X}_{s .}\right)=\prod_{s=1}^{m} \prod_{C \in \mathbf{C}_{s}}\left(1-\prod_{i \in C}\left(1-X_{s i}\right)\right)
$$

may be used to describe the operation of the system: $\phi_{M F M}(\mathbf{X})=1$ if the system works and $\phi_{M F M}(\mathbf{X})=0$ if the system is down. Formula (5) yields the following compact expression for system's reliability:

$$
R_{M F M}=R_{M F M}(\mathbf{q})=E\left(\phi_{M F M}(\mathbf{X})\right)=E\left(\prod_{s=1}^{m} \prod_{C \in \mathbf{C}_{s}}\left(1-\prod_{i \in C}\left(1-X_{s i}\right)\right)\right)
$$

where $\mathbf{q}=\left(q_{s i}\right)_{m \times n}$ is an $m \times n$ matrix. In most cases, the evaluation of $R_{M F M}$ by the above formula is highly facilitated by using the variables $X_{s i}^{\prime}=1-X_{s i}, s \in S, i \in I$ instead of $X_{s i}$ in the expression inside the mean value operator. Taking into account that $\left(X_{s i}^{\prime}\right)^{k}=X_{s i}^{\prime}, k \in\{1,2, \ldots\}$, $X_{s i}^{\prime} X_{t i}^{\prime}=0$ for all $s \neq t, E\left(X_{s i}^{\prime}\right)=q_{s i}$ and

$E\left(\prod_{(s, i) \in A} X_{s i}^{\prime}\right)=\left\{\begin{array}{l}0, \text { if there exist at least two pairs }(s, i),(h, j) \in A \text { with } i=j, s \neq h \\ \prod_{(s, i) \in A} E\left(X_{s i}^{\prime}\right)=\prod_{(s, i) \in A} q_{s i}, \text { otherwise }\end{array}, A \subset S \times I\right.$

(the last outcome results from the independence of $\mathbf{X}_{\cdot i}^{\prime}, \mathbf{X}_{\cdot j}^{\prime}$ for $i \neq j$ ) we may readily arrive, after some lengthy but straightforward algebraic manipulations, at an expression involving sum of products of the form $\prod_{(s, i) \in A} q_{s i}$.

The aforementioned approach can be best illustrated by the following simple example. Consider a consecutive 2, 2-out-of- $n: D F M$ system which fails if and only if at least 2 consecutive components are in the same failure mode (see Koutras [16]). In this case we have $m=2, n=4, \mathbf{C}_{1}=\mathbf{C}_{2}=$ $\{\{1,2\},\{2,3\},\{3,4\}\}$ and applying formula (6) we may write

$$
R_{M F M}=E\left(\phi_{1}\left(\mathbf{X}_{1}\right) \phi_{2}\left(\mathbf{X}_{2}\right)\right)=E\left(\prod_{s=1}^{2} \prod_{i=1}^{3}\left(1-\left(1-X_{s i}\right)\left(1-X_{s, i+1}\right)\right)\right) .
$$


Now, by substitution $\left(X_{s i}^{\prime}=1-X_{s i}, s=1,2\right.$ and $\left.i=1,2,3,4\right)$ and reduction (note that $\left(X_{s i}^{\prime}\right)^{2}=$ $\left.X_{s i}^{\prime}, X_{1 i}^{\prime} X_{2 i}^{\prime}=0\right)$ we have,

$$
\begin{aligned}
R_{M F M}= & E\left(\left(1-X_{11}^{\prime} X_{12}^{\prime}\right)\left(1-X_{12}^{\prime} X_{13}^{\prime}\right)\left(1-X_{13}^{\prime} X_{14}^{\prime}\right)\left(1-X_{21}^{\prime} X_{22}^{\prime}\right)\left(1-X_{22}^{\prime} X_{23}^{\prime}\right)\left(1-X_{23}^{\prime} X_{24}^{\prime}\right)\right) \\
= & 1-q_{11} q_{12}-q_{12} q_{13}+q_{11} q_{12} q_{13}-q_{13} q_{14}+q_{12} q_{13} q_{14}-q_{21} q_{22}+ \\
& +q_{13} q_{14} q_{21} q_{22}-q_{22} q_{23}+q_{21} q_{22} q_{23}-q_{23} q_{24}+q_{11} q_{12} q_{23} q_{24}+q_{22} q_{23} q_{24} .
\end{aligned}
$$

The approach established in formula (6) is fairly general and can be applied for any $M F M$ system. Nevertheless, even for moderate values of $n, m,\left|\mathbf{C}_{s}\right|, s \in S$, it becomes unwieldy because of the computational load involved in the reduction of $\phi_{M F M}(\mathbf{X})$ to a sum of products.

A special case where one can avoid using formula (6) is when there exist no system configurations leading to simultaneous failures in more than one failure modes (see, e.g., Satoh, Sasaki, Yuge and Yanasi [23] or Barlow, Hunter and Proschan [4] for a specific system of this type). Such a condition guarantees that the events $\left[\phi_{s}\left(\mathbf{X}_{s}.\right)=0\right],\left[\phi_{s^{\prime}}\left(\mathbf{X}_{s^{\prime}}\right)=0\right], s \neq s^{\prime}$ are disjoint, and exploiting formulae (5), (6) we may write

$$
R_{M F M}=P\left(\prod_{s=1}^{m} \phi_{s}\left(\mathbf{X}_{s .}\right)=1\right)=1-P\left(\bigcup_{s=1}^{m}\left[\phi_{s}\left(\mathbf{X}_{s}\right)=0\right]\right)=1-\sum_{s=1}^{m} P\left(\phi_{s}\left(\mathbf{X}_{s} .\right)=0\right) ;
$$

recalling next the notation (4) we deduce

$$
R_{M F M}=1-\sum_{s=1}^{m}\left(1-R_{s}\left(\mathbf{q}_{s} .\right)\right)=\sum_{s=1}^{m} R_{s}\left(\mathbf{q}_{s} .\right)-m+1 .
$$

As already stated in Section 1, most of the reliability literature deals with binary systems of binary components (SFM systems). The continuing research interest in such models, has resulted in a great variety of techniques for the reliability assessment of them, either through exact algorithms or by the aid of bounds and approximation formulae. Therefore, it seems reasonable to raise the following question: can we study a $M F M$ system by making advantageous use of the machinery developed for SFM systems? An affirmative answer to this is offered by the next theorem.

Theorem 1 The reliability $R_{M F M}$ of a MFM system with $p_{i}>0, i \in I$ is given by

$$
R_{M F M}=R_{S F M} \cdot\left(\prod_{i=1}^{n} \frac{p_{i}^{m-1}}{\prod_{s=1}^{m}\left(p_{i}+q_{s i}\right)}\right)^{-1}
$$

where $R_{S F M}$ stands for the reliability of a SFM system with

I. $m n$ independent components labelled as $(s, i), s \in S, i \in I$ and respective component unreliabilities $Q_{s i}=q_{s i}\left(p_{i}+q_{s i}\right)^{-1}$,

II. $\sum_{s=1}^{m}\left|\mathbf{C}_{s}\right|+n\left(\begin{array}{c}m \\ 2\end{array}\right)$ cut sets described by

a) $\{(s, i), i \in C\}, C \in \mathbf{C}_{s}$ for all $s \in S$ and b) $\{(s, i),(t, i)\}, s, t \in S, s<t$ for all $i \in I$.

Proof. To start, let us introduce the independent binary random variables $Z_{s i}, s \in S, i \in I$ with $E\left(Z_{s i}\right)=1-Q_{s i}=\frac{p_{i}}{p_{i}+q_{s i}}$. Then, for each $i \in I$, the cut sets $\{(s, i),(t, i)\}, s, t \in S, s<t$ induce a binary structure function of the form

$$
h_{i}\left(\mathbf{z}_{\cdot}\right)=\prod_{s, t \in S: s<t}\left(1-\left(1-z_{s i}\right)\left(1-z_{t i}\right)\right)
$$


where $\mathbf{z} \cdot i=\left(z_{1 i}, z_{2 i}, \ldots, z_{m i}\right)$. Note that, this structure function describes a $S F M$ 2-out-of- $m$ system which fails when at least 2 of its components $(1, i),(2, i), \ldots,(m, i)$ fail. Its reliability is given by

$$
E\left(h_{i}\left(\mathbf{Z}_{\cdot i}\right)\right)=\left(\prod_{s=1}^{m} E\left(Z_{s i}\right)\right)\left(1+\sum_{s=1}^{m} \frac{1-E\left(Z_{s i}\right)}{E\left(Z_{s i}\right)}\right)=\left(\prod_{s=1}^{m} \frac{p_{i}}{p_{i}+q_{s i}}\right) \frac{1}{p_{i}}=\frac{p_{i}^{m-1}}{\prod_{s=1}^{m}\left(p_{i}+q_{s i}\right)} .
$$

Let also $h(\mathbf{z})=\prod_{i=1}^{n} h_{i}\left(\mathbf{z}_{\cdot}\right), \mathbf{z}=\left(z_{s i}\right)_{m \times n}$ and

$$
\epsilon=E(h(\mathbf{Z}))=\prod_{i=1}^{n} E\left(h_{i}\left(\mathbf{Z}_{\cdot i}\right)\right)=\prod_{i=1}^{n} \frac{p_{i}^{m-1}}{\prod_{s=1}^{m}\left(p_{i}+q_{s i}\right)} .
$$

Observing now that the structure function induced by the cut sets II a) equals

$$
\prod_{s=1}^{m} \prod_{C \in \mathbf{C}_{s}}\left(1-\prod_{i \in C}\left(1-z_{s i}\right)\right)=\phi_{M F M}(\mathbf{z})
$$

we may write

$$
\begin{aligned}
R_{S F M} & =E\left(\phi_{M F M}(\mathbf{Z}) h(\mathbf{Z})\right)=\sum_{\mathbf{x} \in\{0,1\}^{m \times n}} \phi_{M F M}(\mathbf{x}) h(\mathbf{x}) P(\mathbf{Z}=\mathbf{x})= \\
& =\epsilon \sum_{\mathbf{x} \in\{0,1\}^{m \times n}} \phi_{M F M}(\mathbf{x}) \prod_{i=1}^{n}\left(\frac{h_{i}\left(\mathbf{x}_{\cdot}\right) P\left(\mathbf{Z}_{\cdot i}=\mathbf{x}_{\cdot}\right)}{E\left(h_{i}\left(\mathbf{Z}_{\cdot i}\right)\right)}\right) .
\end{aligned}
$$

It is not difficult to verify that

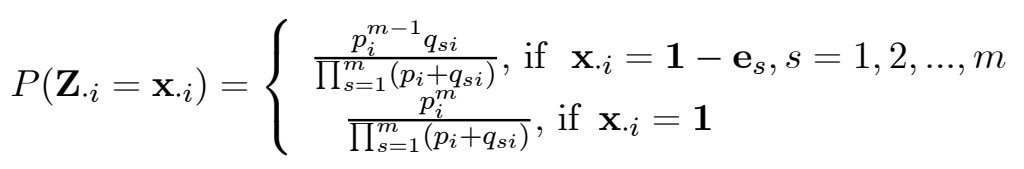

and by virtue of (12) we obtain

$$
h_{i}\left(\mathbf{x}_{\cdot i}\right)=\left\{\begin{array}{l}
1, \text { if } \sum_{s=1}^{m} x_{s i}=m-1 \text { or } m \\
0, \text { if } \sum_{s=1}^{m} x_{s i}<m-1
\end{array} \quad, \frac{h_{i}\left(\mathbf{x}_{\cdot i}\right) P\left(\mathbf{Z}_{\cdot i}=\mathbf{x}_{\cdot i}\right)}{E\left(h\left(\mathbf{Z}_{\cdot}\right)\right)}=\left\{\begin{array}{l}
p_{i}, \text { if } \mathbf{x}_{\cdot i}=\mathbf{1} \\
q_{s i}, \text { if } \mathbf{x}_{\cdot i}=\mathbf{1}-\mathbf{e}_{s} \\
0, \text { otherwise }
\end{array}\right.\right.
$$

The proof is now easily completed if we note that the last expression equals $P\left(\mathbf{X}_{\cdot i}=\mathbf{x} \cdot i\right)$ and substitute in the RHS of (14) to deduce

$$
\begin{aligned}
R_{S F M} & =\epsilon \sum_{\mathbf{x} \in\{0,1\}^{m \times n}} \phi_{M F M}(\mathbf{x}) \prod_{i=1}^{n} P\left(\mathbf{X}_{\cdot i}=\mathbf{x}_{\cdot i}\right) \\
& =\epsilon \sum_{\mathbf{x} \in\{0,1\}^{m \times n}} \phi_{M F M}(\mathbf{x}) P(\mathbf{X}=\mathbf{x})=\epsilon E\left(\phi_{M F M}(\mathbf{X})\right)=\epsilon R_{M F M}
\end{aligned}
$$

As an illustration of the above theorem, let us recalculate the reliability of a consecutive 2,2out-of-4:DFM system by the aid of formula (10). The associated SFM system will consist of 
$m \cdot n=2 \cdot 4=8$ components labelled as $(s, i), s=1,2, \quad i=1,2,3,4$ and its cut sets will be described as $\{(s, i),(s, i+1)\}, s=1,2, i=1,2,3$ and $\{(1, i),(2, i)\}, i=1,2,3,4$.

Let $Z_{s i}$ be independent binary r.v.'s with $E\left(Z_{s i}\right)=1-Q_{s i}=\frac{p_{i}}{p_{i}+q_{s i}}$ and set $Z_{s i}^{\prime}=1-Z_{s i}$. Then, the reliability of the associated $S F M$ system will be given by

$$
\begin{aligned}
R_{S F M}= & E\left(\left(1-Z_{11}^{\prime} Z_{12}^{\prime}\right)\left(1-Z_{12}^{\prime} Z_{13}^{\prime}\right)\left(1-Z_{13}^{\prime} Z_{14}^{\prime}\right) \cdot\left(1-Z_{21}^{\prime} Z_{22}^{\prime}\right)\left(1-Z_{22}^{\prime} Z_{23}^{\prime}\right)\left(1-Z_{23}^{\prime} Z_{24}^{\prime}\right)\right. \\
& \left.\cdot\left(1-Z_{11}^{\prime} Z_{21}^{\prime}\right)\left(1-Z_{12}^{\prime} Z_{22}^{\prime}\right)\left(1-Z_{13}^{\prime} Z_{23}^{\prime}\right)\left(1-Z_{14}^{\prime} Z_{24}^{\prime}\right)\right)
\end{aligned}
$$

and expression (7) can be easily re-established by invoking formula (10).

It is worth mentioning that, due to the presence of $p_{i}^{m-1}$ in the denominator of (10), Theorem 1 requires $p_{i}>0$ for all $i \in I$. Nevertheless, $p_{i}^{m-1}$ are factors of $R_{S F M}$ and therefore the final expression after cancelling these terms, is also valid for the cases where some of the $p_{i}$ 's vanish.

Clearly, the majority of the computational load for the application of Theorem 1 is occupied by the evaluation of the term $R_{S F M}$. Unfortunately, when $n, m,\left|\mathbf{C}_{s}\right|, s \in S$ increase, the size of the associated $S F M$ system and the number of its minimal cut sets will increase so rapidly that the computational complexity of the problem at hand renders the approach as non-feasible. However, as we shall see in the next section, the existence of the compact formula (10) makes it possible to construct useful reliability bounds for $M F M$ systems.

As it is clearly indicated by expression (5), the $M F M$ systems considered so far can be viewed as fictitious systems in which the families of cut sets of type $s$ have been used to formulate certain SFM subsystems and all these subsystems have been replicated and organized in a series structure. Fortunately, the techniques practised in this section can be adapted for use in the more general case where the connection of the subsystems is performed in an arbitrary way.

Let us consider the general case where the $M F M$ system under investigation is described by a structure function of the form

$$
\phi_{M F M}(\mathbf{X})=\psi\left(\phi_{1}\left(\mathbf{X}_{1} \cdot\right), \phi_{2}\left(\mathbf{X}_{2 .}\right), \ldots, \phi_{m}\left(\mathbf{X}_{m} \cdot\right)\right)
$$

where $\psi:\{0,1\}^{m} \rightarrow\{0,1\}$ is a coordinatewise nondecreasing function. The binary function $\psi$ describes the way our fictitious subsystems are organized and is itself a structure function of a $S F M$ system with $m$ components. Denoting by $\boldsymbol{\Gamma}$ the family of minimal cut sets associated with $\psi$, we may express the reliability $R_{M F M}$ of the extended $M F M$ system as

$$
R_{M F M}=E\left(\phi_{M F M}(\mathbf{X})\right)=E\left(\prod_{\Gamma \in \boldsymbol{\Gamma}}\left(1-\prod_{s \in \Gamma}\left(1-\phi_{s}\left(\mathbf{X}_{s}\right)\right)\right)\right) .
$$

Apparently for $\boldsymbol{\Gamma}=\{\{1\},\{2\}, \ldots,\{m\}\}$ the $M F M$ systems studied earlier pop up.

By way of example let us consider the special case of a DFM system $(m=2)$ with 5 independent components, cut set families $\mathbf{C}_{1}=\mathbf{C}_{2}=\{\{1,2\},\{2,3\},\{3,4\},\{4,5\}\}$ and $\boldsymbol{\Gamma}=\{\{1,2\}\}$ (i.e., $\psi\left(y_{1}, y_{2}\right)=1-\left(1-y_{1}\right)\left(1-y_{2}\right)$ is the structure function of a $S F M$ parallel system with two components). Apparently,

$$
\phi_{s}\left(\mathbf{X}_{s .}\right)=\prod_{i=1}^{4}\left(1-\left(1-X_{s i}\right)\left(1-X_{s, i+1}\right)\right)=\prod_{i=1}^{4}\left(1-X_{s i}^{\prime} X_{s, i+1}^{\prime}\right), s=1,2
$$


and by the aid of (17) $R_{M F M}$ can be expressed as

$$
\begin{aligned}
R_{M F M}= & E\left(1-\left(1-\phi_{1}\left(\mathbf{X}_{1 .}\right)\right)\left(1-\phi_{2}\left(\mathbf{X}_{2 .}\right)\right)\right) \\
= & 1-q_{13} q_{14} q_{21} q_{22}-q_{14} q_{15} q_{21} q_{22}+q_{13} q_{14} q_{15} q_{21} q_{22}-q_{14} q_{15} q_{22} q_{23}+q_{14} q_{15} q_{21} q_{22} q_{23} \\
& -q_{11} q_{12} q_{23} q_{24}-q_{11} q_{12} q_{24} q_{25}-q_{12} q_{13} q_{24} q_{25}+q_{11} q_{12} q_{13} q_{24} q_{25}+q_{11} q_{12} q_{23} q_{24} q_{25}
\end{aligned}
$$

Let us now return to the general case of an extended MFM system and investigate how Theorem 1 could be modified in order to accommodate the new framework. Denote by $Z_{s i}, s \in S, i \in I$ a set of $m n$ independent binary r.v.'s with failure probabilities $Q_{s i}=q_{s i}\left(p_{i}+q_{s i}\right)^{-1}$ and by $h_{i}(\cdot)$ the structure function of a $S F M$ 2-out-of- $n$ system (cf. (11)). Following the technique indicated in the proof of Theorem 1 we may easily verify that $R_{M F M}$ can again be evaluated via formula (10), with $R_{S F M}$ being given by

$$
R_{S F M}=E\left(\psi\left(\phi_{1}\left(\mathbf{Z}_{1 \cdot}\right), \ldots, \phi_{m}\left(\mathbf{Z}_{m}\right)\right) \prod_{i=1}^{n} h_{i}\left(\mathbf{Z}_{\cdot i}\right)\right) .
$$

Although some of the results established in the next sections could be stated for a general $M F M$ system as described by (16), in what follows we shall abandon this set-up for typographical convenience and proceed to the investigation of the original MFM system introduced in Section 1.

\section{Reliability Bounds}

In this section we exploit the exact reliability formulae developed in Section 3 in order to establish reliability bounds for $M F M$ systems.

Observe first that, by virtue of (8), the following lower (Bonferroni) bound ensues

$$
R_{M F M}=1-P\left(\bigcup_{s=1}^{m}\left[\phi_{s}\left(\mathbf{X}_{s .}\right)=0\right]\right) \geq 1-\sum_{s=1}^{m}\left(1-R_{s}\left(\mathbf{q}_{s .}\right)\right)=\sum_{s=1}^{m} R_{s}\left(\mathbf{q}_{s .}\right)-m+1 \equiv L_{\text {Bonf }} .
$$

The derivation of the rest of the bounds we shall deal with, call for the use of the theory of negatively associated (NA) random variables. (cf. Joag-Dev and Proschan [15]). Recently, Boutsikas and Koutras [6] proved that, if $Z_{1}, Z_{2}, \ldots, Z_{k}$ are $N A$ non-negative, integer valued r.v.'s, such that $E\left(Z_{i}\right), E\left(Z_{i} Z_{j}\right)<\infty, i, j=1,2, \ldots, k, i \neq j$, then

$$
0 \leq \prod_{i=1}^{k} P\left(Z_{i}=0\right)-P\left(Z_{i}=0, i=1,2, \ldots, k\right) \leq-\sum_{i<j} \operatorname{Cov}\left(Z_{i}, Z_{j}\right) .
$$

For the special case of binary $N A$ r.v.'s $Z_{i}, i=1,2, \ldots, k$ the above inequality yields (note that $1-Z_{i}, i=1,2, \ldots, k$ are also $\mathrm{NA}$ )

$$
0 \leq \prod_{i=1}^{k} E\left(Z_{i}\right)-E\left(\prod_{i=1}^{k} Z_{i}\right) \leq-\sum_{i<j} \operatorname{Cov}\left(Z_{i}, Z_{j}\right)
$$

A key observation for the development of our bounds is that the binary random matrix $\mathbf{X}=\left(X_{s i}\right)$ consists of $N A$ random variables. This is proved in the next lemma. 
Lemma 1 The mn binary random variables $X_{\text {si }}, s \in S, i \in I$ are $N A$.

Proof. It is obvious that the $m+1$-dimensional binary random variable $\left(\prod_{s=1}^{m} X_{s i}, \mathbf{1}-\mathbf{X}_{. i}\right)$ follows a multinomial distribution with parameters 1 and $\left(p_{i}, q_{1 i}, \ldots, q_{m i}\right)$. As Joag-Dev and Proschan [15] have proved, a collection of r.v.'s obeying the multinomial law is NA; therefore the set of r.v.'s $\left\{\prod_{s=1}^{m} X_{s i}, 1-X_{1 i}, \ldots, 1-X_{m i}\right\}$ is $N A$ and the same will hold true for its subset $\left\{1-X_{1 i}, \ldots, 1-X_{m i}\right\}$. The last conclusion guarantees that all the sets $\left\{X_{1 i}, \ldots, X_{m i}\right\}, i \in I$ are $N A$ and the proof is completed by recalling that the $N A$ property is preserved when considering the union of independent $N A$ sets.

We are now in possession of all necessary machinery to establish reliability bounds of $M F M$ systems by exploiting the exact formulae (6) and (10).

Theorem 2 The reliability $R_{M F M}$ of a MFM system is bounded below and above as follows

$$
\prod_{s=1}^{m} R_{s}\left(\mathbf{q}_{s} .\right)-\sum_{s<t} \sum_{C \in \mathbf{C}_{s}} \sum_{\substack{D \in \mathbf{C}_{t}: i \in C \\ D \cap C \neq \emptyset}} \prod_{i \in C} q_{s i} \prod_{j \in D} q_{t j} \leq R_{M F M} \leq \prod_{s=1}^{m} R_{s}\left(\mathbf{q}_{s} .\right)
$$

Proof. By Lemma 1, the binary r.v.'s $X_{s i}, s \in S, i \in I$ are $N A$ and the same will hold true for $\phi_{s}\left(\mathbf{X}_{s}\right), s \in S$ which are nondecreasing functions defined over disjoined sets of $\left\{X_{s i}, s \in S, i \in I\right\}$. A direct application of the covariance inequality (20) yields

$$
0 \leq \prod_{s=1}^{m} E\left(\phi_{s}\left(\mathbf{X}_{s .}\right)\right)-E\left(\prod_{s=1}^{m} \phi_{s}\left(\mathbf{X}_{s}\right)\right) \leq-\sum_{s<t} \operatorname{Cov}\left(\phi_{s}\left(\mathbf{X}_{s}\right), \phi_{t}\left(\mathbf{X}_{t} .\right)\right)
$$

and employing formulae (4) and (5), we may write the inequality in the more interesting form

$$
\prod_{s=1}^{m} R_{s}\left(\mathbf{q}_{s .}\right)+\sum_{s<t} \operatorname{Cov}\left(\phi_{s}\left(\mathbf{X}_{s .}\right), \phi_{t}\left(\mathbf{X}_{t} .\right)\right) \leq R_{M F M} \leq \prod_{s=1}^{m} R_{s}\left(\mathbf{q}_{s .}\right) .
$$

Let us next introduce the integer valued r.v.'s $H_{s}=\sum_{C \in \mathbf{C}_{s}} \prod_{i \in C}\left(1-X_{s i}\right), s \in S$ which are $N A$ and vanish if and only if $\phi_{s}\left(\mathbf{X}_{s}\right.$.) equals 1 . Applying (19) for $k=2$ we obtain

$$
P\left(H_{s}=0\right) P\left(H_{t}=0\right)-P\left(H_{s}=0, H_{t}=0\right) \leq-\operatorname{Cov}\left(H_{s}, H_{t}\right), s<t
$$

and recognizing that the LHS equals $-\operatorname{Cov}\left(\phi_{s}\left(\mathbf{X}_{s}\right), \phi_{t}\left(\mathbf{X}_{t}\right)\right)$, we may restate the last inequality as

$$
-\operatorname{Cov}\left(\phi_{s}\left(\mathbf{X}_{s}\right), \phi_{t}\left(\mathbf{X}_{t} \cdot\right)\right) \leq-\operatorname{Cov}\left(\sum_{C \in \mathbf{C}_{s}} \prod_{i \in C}\left(1-X_{s i}\right), \sum_{D \in \mathbf{C}_{t}} \prod_{j \in D}\left(1-X_{t j}\right)\right), s<t .
$$

The proof is now easily completed if we take into account that

$$
\operatorname{Cov}\left(\prod_{i \in C}\left(1-X_{s i}\right), \prod_{j \in D}\left(1-X_{t j}\right)\right)=\left\{\begin{array}{c}
0 \text { if } C \cap D=\emptyset \\
-\prod_{i \in C} q_{s i} \prod_{j \in D} q_{t j} \text { if } C \cap D \neq \emptyset
\end{array}\right.
$$


In the sequel, the upper and lower bounds of Theorem 2 will be denoted by $U, L$ respectively, i.e.,

$$
U=\prod_{s=1}^{m} R_{s}\left(\mathbf{q}_{s}\right), \quad L=U-\sum_{s<t} c_{s t}, \text { where } c_{s t}=\sum_{C \in \mathbf{C}_{s}} \sum_{\substack{D \in \mathbf{C}_{t}: \\ D \cap C \neq \emptyset}} \prod_{i \in C} q_{s i} \prod_{j \in D} q_{t j} .
$$

It is worth mentioning than formula (22) induces the following additional lower bound which deserves special attention

$$
\prod_{s=1}^{m} R_{s}\left(\mathbf{q}_{s .}\right)-\sum_{s<t}\left(R_{s}\left(\mathbf{q}_{s .}\right) R_{t}\left(\mathbf{q}_{t} .\right)-E\left(\phi_{s}\left(\mathbf{X}_{s}\right) \phi_{t}\left(\mathbf{X}_{t} .\right)\right)\right) \leq R_{M F M} .
$$

The last bound is better that $L$, but in most cases requires much more complicated effort, because of the presence of the terms $E\left(\phi_{s}\left(\mathbf{X}_{s} \cdot\right) \phi_{t}\left(\mathbf{X}_{t}\right)\right)$.

A further point of interest is that the distance $d=U-L_{B o n f}$ between the upper bound of Theorem 2 and the Bonferroni lower bound (18), can be bounded above as follows

$$
d=U-L_{\text {Bonf }} \leq \sum_{r<s}\left(1-R_{r}\left(\mathbf{q}_{r} .\right)\right)\left(1-R_{s}\left(\mathbf{q}_{s .}\right)\right) \leq\left(\begin{array}{c}
m \\
2
\end{array}\right)\left(1-R_{M F M}\right)^{2} \leq \frac{\left(m\left(1-R_{M F M}\right)\right)^{2}}{2}
$$

The second inequality results from the fact that $R_{M F M} \leq R_{s}\left(\mathbf{q}_{s .}\right), s=1,2, \ldots, m$ (cf. (21)), while the first one is readily ascertainable from the classical Bonferroni inequality for independent events

$$
1-\prod_{s=1}^{m} P\left(A_{s}\right)=P\left(\bigcup_{s=1}^{m} A_{s}^{c}\right) \geq \sum_{s=1}^{m} P\left(A_{s}^{c}\right)-\sum_{r<s} P\left(A_{r}^{c}\right) P\left(A_{s}^{c}\right)
$$

Inequality (25) reveals that, the combined use of $L_{B o n f}$ and $U$ for highly reliable systems $\left(R_{M F M}\right.$ close to 1 ), will provide very accurate interval estimation for system's reliability. For example, if $m\left(1-R_{M F M}\right)<0.2$ then the interval length is at most $\frac{1}{2}\left(m\left(1-R_{M F M}\right)\right)^{2}=2 \%$.

Although a mathematical comparison between $L$ and $L_{B o n f}$ does not seem feasible, our extensive numerical experimentation revealed that usually the performance of $L$ is superior to that of $L_{\text {Bonf }}$ (more details on this can be found in Section 6).

We are now going to state an additional lower bound for $M F M$ systems. Its derivation will be couched on the $S F M$ representation of an $M F M$ system as described in Theorem 1.

Theorem 3 Let $\mathbf{Q}=\left(Q_{s i}\right)$ with $Q_{s i}=q_{s i}\left(p_{i}+q_{s i}\right)^{-1}<1, s \in S, i \in I$ and denote by $\mathbf{Q}_{s} .=$ $\left(Q_{s 1}, \ldots, Q_{s n}\right)$ the rows of matrix $\mathbf{Q}$. Then, the reliability $R_{M F M}$ of the MFM system is bounded below by the quantity

$$
L_{S F M}=\prod_{s=1}^{m} R_{s}\left(\mathbf{Q}_{s}\right)
$$

Proof. Adhering to the notations used in the proof of Theorem 1 we may write

$$
R_{M F M}=\frac{R_{S F M}}{\epsilon}=\frac{E\left(\phi_{M F M}(\mathbf{Z}) h(\mathbf{Z})\right)}{E(h(\mathbf{Z}))} .
$$


Since $Z_{s i}, s \in S, i \in I$ are associated (as a collection of independent r.v.'s), it follows that their coordinatewise nondecreasing functions $\phi_{M F M}(\mathbf{Z}), h(\mathbf{Z})$ will be associated as well, and therefore the next inequality will hold true (see e.g. Esary, Proschan and Walkup [12])

$$
E\left(\phi_{M F M}(\mathbf{Z}) h(\mathbf{Z})\right) \geq E\left(\phi_{M F M}(\mathbf{Z})\right) E(h(\mathbf{Z})) .
$$

Hence $R_{M F M} \geq E\left(\phi_{M F M}(\mathbf{Z})\right)$ and the desired result is easily obtained by restating $\phi_{M F M}(\mathbf{Z})$ in the form (cf. (5))

$$
E\left(\phi_{M F M}(\mathbf{Z})\right)=\prod_{s=1}^{m} E\left(\phi_{s}\left(\mathbf{Z}_{s}\right)\right)=\prod_{s=1}^{m} R_{s}\left(\mathbf{Q}_{s}\right) .
$$

As our numerical experimentation revealed (see Section 6) none of the two lower bounds $L_{B o n f}, L_{S F M}$ is uniformly better than the other. However, for large systems, $L_{S F M}$ seems to behave significantly better than $L_{B o n f}$; As a matter of fact, a sophisticated use of $L_{S F M}$ along with the upper bound of Theorem 2 will enable us to state several interesting asymptotic results.

\section{The reliability of large $M F M$ systems}

The investigation of the asymptotic behaviour of reliability structures usually offers quite interesting insights to the performance of large systems, i.e., systems with large number of components. On the other hand, the fact that most real life applications call for the study of huge reliability structures (usually with high quality components), makes the asymptotic studies significant, not only from the theoretical point of view but for practical applications as well.

The problem we are focusing on in this section can be summarized in the following question: given a specific type of $M F M$ systems and a procedure to increase the number of its components without bound, what are the appropriate conditions such that the limiting system reliability is non-degenerate? To achieve this goal, we are going to make beneficial use of the bounds developed in the previous section.

We begin by introducing first some additional notations. Denote by $I_{\nu}$ the set of components of the $\nu$-th system, by $\{0\} \cup S_{\nu}=\left\{0,1, \ldots, m_{\nu}\right\}$ the state space for the components $i \in I_{\nu}$ and by $\mathbf{C}_{s}(\nu)$ the associated sequence of families of minimal cut sets of type $s, s \in S_{\nu}$. The survival probability of component $i \in I_{\nu}$ will be denoted by $p_{i}(\nu)$ while the probability that component $i \in I_{\nu}$ is in state $s \in S_{\nu}$ by $q_{s i}(\nu)$.

We also set

$$
\begin{aligned}
p(\nu) & =\min _{i \in I_{\nu}} p_{i}(\nu), \quad \mu_{s}(\nu)=\sum_{C \in \mathbf{C}_{s}(\nu)} \prod_{i \in C} q_{s i}(\nu), \quad q_{s}(\nu)=\max _{i \in I_{\nu}} q_{s i}(\nu), \\
d_{s}(\nu) & =\min _{C \in \mathbf{C}_{s}(\nu)}|C|, \quad c_{s}(\nu)=\max _{C \in \mathbf{C}_{s}(\nu)}|C|, \quad s \in S_{\nu} \\
q(\nu) & =\max _{s \in S_{\nu}} q_{s}(\nu), \quad d(\nu)=\min _{s \in S_{\nu}} d_{s}(\nu)
\end{aligned}
$$

and

$$
a_{s t}(\nu)=\max _{C \in \mathbf{C}_{s}(\nu)}\left|\left\{D \in \mathbf{C}_{t}(\nu): C \cap D \neq \emptyset\right\}\right|, s, t \in S_{\nu}, s \neq t, \quad a(\nu)=\max _{s, t \in S_{\nu}, s \neq t} a_{s t}(\nu) .
$$


(For the evaluation of $a_{s t}(\nu)$ we consider first the cardinality of the set $\left|\left\{D \in \mathbf{C}_{t}(\nu): C \cap D \neq \emptyset\right\}\right|$ of all the elements $D \in \mathbf{C}_{t}(\nu)$ that intersect with a set $C \in \mathbf{C}_{s}(\nu)$ and then the maximum cardinality for all $C \in \mathbf{C}_{s}(\nu)$ is captured). Making use of the above notations we may easily verify that the subtractor of the LHS in (21) can be bounded above by

$$
\sum_{s<t} \sum_{C \in \mathbf{C}_{s}(\nu)} \prod_{i \in C} q_{s i}(\nu) \sum_{\substack{D \in \mathbf{C}_{t}: \\ C \cap D \neq \emptyset}} q_{t}(\nu)^{d_{t}(\nu)} \leq \sum_{t=1}^{m_{\nu}} \sum_{s=1}^{t-1} \mu_{s}(\nu) a_{s t}(\nu) q_{t}(\nu)^{d_{t}(\nu)} \leq m_{\nu} a(\nu) q(\nu)^{d(\nu)} \sum_{s=1}^{m_{\nu}} \mu_{s}(\nu)
$$

Therefore, in view of Theorem 2, if the last bound vanishes as $\nu \rightarrow \infty$, then the reliability $R_{M F M}(\nu)$ of the $\nu$-th $M F M$ system can be approximated by the product $\prod_{s=1}^{m_{\nu}} R_{s \nu}\left(\mathbf{q}_{s} \cdot(\nu)\right)\left(R_{s \nu}\left(\mathbf{q}_{s .}(\nu)\right)\right.$ is the reliability of a $S F M$ system with components $i \in I_{\nu}$, minimal cut sets $C \in \mathbf{C}_{s}(\nu)$ and component failure probabilities $\left.q_{s i}(\nu), i \in I_{\nu}\right)$. For example, should the number $m_{\nu}$ of failure modes, the expected number $\sum_{s=1}^{m_{\nu}} \mu_{s}(\nu)$ of failed minimal cut sets and the maximum overlap $a(\nu)$ between minimal cut sets be bounded, the following result holds true

$$
R_{M F M}(\nu)=\prod_{s=1}^{m_{\nu}} R_{s \nu}\left(\mathbf{q}_{s \cdot}(\nu)\right)+O\left(q(\nu)^{d(\nu)}\right)
$$

One could further proceed to built up specific conditions guaranteeing the convergence of upper bound (27) to 0 and the simultaneous convergence of $\prod_{s=1}^{m_{\nu}} R_{s \nu}\left(\mathbf{q}_{s} \cdot(\nu)\right)$ to a finite limit. However, for the investigation of the asymptotic behaviour of MFM systems, it seems much more efficient to make a combined use of the upper bound in (21) and the lower bound (26) which are simple multiplicative bounds referring to SFM systems with the same structure function and different component failure probabilities, namely

$$
q_{s i}(\nu), i \in I_{\nu} \text { and } Q_{s i}(\nu)=\frac{q_{s i}(\nu)}{p_{i}(\nu)+q_{s i}(\nu)}, i \in I_{\nu}
$$

Our asymptotic results for $M F M$ systems will be accomplished by appealing to a limit theorem that was recently proved by $\mathrm{Fu}$ and Koutras [13] for $S F M$ systems. If $\mathbf{C}(\nu)=\left\{C_{1}, C_{2}, \ldots, C_{N(\nu)}\right\}$ is the family of minimal cut sets of the $\nu$-th $S F M$ system and $I_{\nu}$ its set of components, one can always choose a collection of index sets $L_{1}=\emptyset$ and $L_{j} \subset I_{\nu}, j=2,3, \ldots, N(\nu)$ such that

$$
L_{j} \cap C_{j}=\emptyset \text { and } L_{j} \cap C \neq \emptyset \text { for all } C \in\left\{C_{i}, i=1,2, \ldots, j-1: C_{i} \cap C_{j} \neq \emptyset\right\} .
$$

(note that the index $\nu$ has been suppressed in the minimal cut sets $C_{j}$ and index sets $L_{j}$ ). If $q_{i}(\nu), i \in I_{\nu}$ are the failure probabilities of the components in the $\nu$-th system, the next limit theorem holds true for system's reliability $R(\nu)$ (cf. Fu and Koutras [13]).

Theorem 4 If $\lim _{\nu \rightarrow \infty} N(\nu)=\infty$ such that

$$
\lim _{\nu \rightarrow \infty} \sum_{j=1}^{N(\nu)} \prod_{i \in C_{j}(\nu)} q_{i}(\nu)=\lambda \text { and } \lim _{\nu \rightarrow \infty} \max \left\{1, \max _{1 \leq j \leq N(\nu)}\left|L_{j}\right|\right\} \cdot \max _{i \in I_{\nu}} q_{i}(\nu)=0 .
$$

then $\lim _{\nu \rightarrow \infty} R(\nu)=e^{-\lambda}$. 
Let us now assume that the members of the $\nu$-th family of minimal cut sets of type $s$ have been labelled as $\mathbf{C}_{s}(\nu)=\left\{C_{s 1}, C_{s 2}, \ldots, C_{s, N_{s}(\nu)}\right\}$ and set $l_{s}(\nu)=\max \left\{1, \max _{1 \leq j \leq N_{s}(\nu)}\left|L_{s j}\right|\right\}$, where $L_{s j}, j=1,2, \ldots, N_{s}(\nu)$ are collections of index sets satisfying (29) for the family $\mathbf{C}_{s}(\nu)$. Should the number $m_{\nu}$ of failure modes be kept constant as $\nu \rightarrow \infty$, the asymptotic behaviour of the MFM system can be described by the next theorem.

Theorem 5 If $\lim _{\nu \rightarrow \infty} N_{s}(\nu)=\infty$ and

$$
\text { (a) } \lim _{\nu \rightarrow \infty} \sum_{j=1}^{N_{s}(\nu)} \prod_{i \in C_{s j}(\nu)} q_{s i}(\nu)=\lambda_{s}, \quad(b) \lim _{\nu \rightarrow \infty} l_{s}(\nu) q_{s}(\nu)=0, \quad(c) \lim _{\nu \rightarrow \infty} c_{s}(\nu)(1-p(\nu))=0
$$

for every $s=1,2, \ldots, m$, then $\lim _{\nu \rightarrow \infty} R_{M F M}(\nu)=e^{-\left(\lambda_{1}+\lambda_{2}+\ldots+\lambda_{m}\right)}$.

Proof. Bounds (23), (26) take on the form

$$
U(\nu)=\prod_{s=1}^{m} R_{s \nu}\left(\mathbf{q}_{s \cdot}(\nu)\right), \quad L_{S F M}(\nu)=\prod_{s=1}^{m} R_{s \nu}\left(\mathbf{Q}_{s \cdot}(\nu)\right)
$$

where $\mathbf{q}_{s .}(\nu)=\left(q_{s i}(\nu): i \in I_{\nu}\right), \mathbf{Q}_{s} .(\nu)=\left(Q_{s i}(\nu): i \in I_{\nu}\right)$ (cf. (28)). Conditions (a)-(c) allow the application of Theorem 4 for $S F M$ systems with component failure probabilities $q_{s i}(\nu), i \in I_{\nu}$; thus we get $\lim _{\nu \rightarrow \infty} R_{s \nu}\left(\mathbf{q}_{s .}(\nu)\right)=e^{-\lambda_{s}}$ for all $s=1,2, \ldots, m$ and therefore

$$
\lim _{\nu \rightarrow \infty} U(\nu)=e^{-\left(\lambda_{1}+\lambda_{2}+\ldots+\lambda_{m}\right)} .
$$

We shall now use again Theorem 4 to verify that $L_{S F M}(\nu)$ converges to the same limit. To achieve this it suffices to prove that

$$
\text { (i) } \lim _{\nu \rightarrow \infty} \sum_{j=1}^{N_{s}(\nu)} \prod_{i \in C_{s j}(\nu)} Q_{s i}(\nu)=\lambda_{s}, \quad(\text { ii }) \lim _{\nu \rightarrow \infty} l_{s}(\nu) \cdot \max _{i \in I_{\nu}} Q_{s i}(\nu)=0
$$

for all $s=1,2, \ldots, m$. The limiting expression (ii) is easily established by the aid of the inequality

$$
l_{s}(\nu) \cdot \max _{i \in I_{\nu}} \frac{q_{s i}(\nu)}{p_{i}(\nu)+q_{s i}(\nu)} \leq l_{s}(\nu) q_{s}(\nu) \frac{1}{p(\nu)}
$$

and the fact that $\lim _{\nu \rightarrow \infty} l_{s}(\nu) q_{s}(\nu)=0$ and $\lim _{\nu \rightarrow \infty} p(\nu)=1$ (note that the last equality implies the validity of condition $Q_{s i}(\nu)<1$ for infinitely many $\nu$ which is a prerequisite for using the upper bound $L_{S F M}(\nu)$ ). To derive (i), begin by bounding the terms of interest as follows

$$
\begin{aligned}
& \sum_{j=1}^{N_{s}(\nu)} \prod_{i \in C_{s j}(\nu)} Q_{s i}(\nu)=\sum_{j=1}^{N_{s}(\nu)} \prod_{i \in C_{s j}(\nu)} \frac{q_{s i}(\nu)}{p_{i}(\nu)+q_{s i}(\nu)} \geq \sum_{j=1}^{N_{s}(\nu)} \prod_{i \in C_{s j}(\nu)} q_{s i}(\nu), \\
& \sum_{j=1}^{N_{s}(\nu)} \prod_{i \in C_{s j}(\nu)} Q_{s i}(\nu) \leq \sum_{j=1}^{N_{s}(\nu)} \prod_{i \in C_{s j}(\nu)} \frac{q_{s i}(\nu)}{p_{i}(\nu)} \leq \frac{1}{p(\nu)^{c_{s}(\nu)}} \sum_{j=1}^{N_{s}(\nu)} \prod_{i \in C_{s j}(\nu)} q_{s i}(\nu)
\end{aligned}
$$


and use the obvious inequality $\left|1-p(\nu)^{c_{s}(\nu)}\right| \leq c_{s}(\nu)(1-p(\nu))$ and condition (c) to verify that $\lim _{\nu \rightarrow \infty} p(\nu)^{c_{s}(\nu)}=1$. Limiting expression (i) is now effortlessly deduced by observing that, by virtue of condition (a), both aforementioned bounds converge to $\lambda_{s}$. This completes the proof.

Alert readers may see that conditions (b) and (c) of Theorem 5 could be replaced by the single condition $\lim _{\nu \rightarrow \infty} l_{s}(\nu) c_{s}(\nu)(1-p(\nu))=0\left(\right.$ note that $l_{s}(\nu) c_{s}(\nu)(1-p(\nu)) \geq \max \left\{l_{s}(\nu) q_{s}(\nu), c_{s}(\nu)(1-\right.$ $p(\nu))\}$. In most practical applications the sequences $l_{s}(\nu), c_{s}(\nu)$ are bounded and, therefore in such cases, the only assumption needed is $\lim _{\nu \rightarrow \infty} p(\nu)=1$.

\section{Applications}

Several single failure mode systems that have recently received considerable research attention can be extended in a very natural way to $M F M$ systems. For example, the well known $k$-outof- $n$, consecutive $k$-out-of- $n, r$-within-consecutive $k$-out-of- $n$ systems and their two dimensional analogues can be effortlessly adjusted to a multifailure environment and subsequently investigated by the use of the techniques presented in the previous section. In this section we shall restrict ourselves to the application of our general bounds and asymptotic results to two $M F M$ reliability structures that have resulted by considering appropriate extensions of the classical $k$-out-of- $n$ and consecutive- $k$-out-of- $n$ systems. For typographical convenience, throughout this section we shall be assuming that the components are identical (iid case) with $p_{i}=p, q_{s i}=q_{s}, s \in S$ for all $i \in I$.

\section{a) consecutive $k_{1}, k_{2}, \ldots, k_{m}$-out-of- $n$ :MFM system}

A consecutive $k_{1}, k_{2}, \ldots, k_{m}$-out-of- $n$ MFM system consists of $n$ linearly arranged components and enters failure mode $s$ whenever at least $k_{s}$ consecutive components are failed in mode $s$, $s=1,2, \ldots, m$. The consecutive- $k_{1}, k_{2}$-out-of- $n$ :DFM systems $(m=2)$ were recently studied by Koutras [16]. In Section 3 we used the special case $n=4, k_{1}=k_{2}=2, m=2$ to illustrate the exact reliability evaluation techniques developed there. Chryssaphinou and Vaggelatou [9] exploited the celebrated Stein-Chen method to establish reliability bounds for a consecutive- $k_{1}, \ldots, k_{m}$-out-of$n: M F M$ system with Markov dependent components.

It is of interest to note that the reliability of the system is associated with a class of run waiting time problems in a sequence of discrete random variables, Aki [1]. The special case $m=2$ leads to sooner waiting time problems in sequences of trinary outcomes; see Koutras and Alexandrou [17] for exact formulae through a Markov chain imbedding approach and Stein-Chen bounds.

Let $I=\{1,2, \ldots, n\}$ denote the set of components of a consecutive $k_{1}, k_{2}, \ldots, k_{m}$-out-of- $n M F M$ system. Then its minimal cut sets of type $s$ will be given by $\mathbf{C}_{s}=\left\{\left\{i, i+1, \ldots, i+k_{s}-1\right\}, i=\right.$ $\left.1,2, \ldots, n-k_{s}+1\right\}, s=1,2, \ldots, m$. Denoting by $R_{s}(q)$ the reliability of an ordinary iid $S F M$ consecutive $k_{s}$-out-of- $n$ system with component unreliabilities $q$ we may deduce the following reliability bounds for consecutive $k_{1}, k_{2}, \ldots, k_{m}$-out-of- $n$ systems (cf. (18), (23) and (26))

$$
\begin{aligned}
& L_{\text {Bonf }}=\sum_{s=1}^{m} R_{s}\left(q_{s}\right)-m+1, \quad L=\prod_{s=1}^{m} R_{s}\left(q_{s}\right)-\sum_{s<t} c_{s t}, \quad U=\prod_{s=1}^{m} R_{s}\left(q_{s}\right), \\
& L_{S F M}=\prod_{s=1}^{m} R_{s}\left(Q_{s}\right), \quad c_{s t} \leq\left(n-k_{s}+1\right)\left(k_{s}+k_{t}-1\right) q_{s}^{k_{s}} q_{t}^{k_{t}}, \quad Q_{s}=\frac{q_{s}}{p+q_{s}}, s=1,2, \ldots, m .
\end{aligned}
$$




\begin{tabular}{cccccccccccc}
\hline$p$ & $q_{1}$ & $q_{2}$ & $q_{3}$ & $L_{S F M}^{\prime}$ & $L^{\prime}$ & $L_{\text {Bonf }}^{\prime}$ & $L_{\text {Bonf }}$ & $L$ & $L_{S F M}$ & $U^{\prime}$ & $U$ \\
\hline 0.3 & 0.35 & 0.2333 & 0.1167 & 0.0007 & 0.2345 & 0.0359 & 0.2048 & 0.3183 & 0.0073 & 0.4068 & 0.3961 \\
0.4 & 0.3 & 0.2 & 0.1 & 0.0421 & 0.4487 & 0.3486 & 0.4491 & 0.5119 & 0.0934 & 0.5485 & 0.5423 \\
0.5 & 0.25 & 0.1667 & 0.0833 & 0.2530 & 0.6353 & 0.5949 & 0.6463 & 0.6741 & 0.3285 & 0.6873 & 0.6843 \\
0.6 & 0.2 & 0.1333 & 0.0667 & 0.5678 & 0.7848 & 0.7722 & 0.7943 & 0.8037 & 0.6126 & 0.8076 & 0.8065 \\
0.7 & 0.15 & 0.1 & 0.05 & 0.8105 & 0.8915 & 0.8888 & 0.8963 & 0.8985 & 0.8253 & 0.8993 & 0.8990 \\
0.8 & 0.1 & 0.0667 & 0.0333 & 0.9387 & 0.9576 & 0.9573 & 0.9590 & 0.9593 & 0.9415 & 0.9594 & 0.9594 \\
0.9 & 0.05 & 0.0333 & 0.0167 & 0.9890 & 0.9908 & 0.9908 & 0.9909 & 0.9909 & 0.9892 & 0.9909 & 0.9909 \\
\hline
\end{tabular}

Table 1: Reliability bounds for consecutive-4,3,2-out-of-30 : MFM systems

The evaluation of $R_{s}\left(q_{s}\right), R_{s}\left(Q_{s}\right)$ can be easily performed by initiating simple recursive schemes, or by using an appropriate Markov chain imbedding approach or even by exact combinatorial formulae; for a review see Chao, Fu and Koutras [8]. Nonetheless, one could take advantage of the availability of simple reliability bounds for $S F M$ consecutive $k$-out-of- $n$ systems and write down several bounds for consecutive- $k_{1}, \ldots, k_{m}$-out-of- $n: M F M$ systems which are quite appealing both in terms of accuracy and computational simplicity. In particular, exploiting the Esary and Proschan [11] and Fu and Koutras [13] bounds (see also Boutsikas and Koutras [7]) we may easily get the following classes of reliability bounds for the $M F M$ system

$$
\begin{aligned}
L_{\text {Bonf }}^{\prime} & =\sum_{s=1}^{m}\left(1-q_{s}^{k_{s}}\right)^{n-k_{s}+1}-m+1, \quad U^{\prime}=\prod_{s=1}^{m}\left(1-q_{s}^{k_{s}}\right)\left(1-\left(1-q_{s}\right) q_{s}^{k_{s}}\right)^{n-k_{s}} \\
L^{\prime} & =\prod_{s=1}^{m}\left(1-q_{s}^{k_{s}}\right)^{n-k_{s}+1}-\sum_{1 \leq s<t \leq m}\left(n-k_{s}+1\right)\left(k_{s}+k_{t}-1\right) q_{s}^{k_{s}} q_{t}^{k_{t}}, \\
L_{S F M}^{\prime} & =\prod_{s=1}^{m}\left(1-\left(\frac{q_{s}}{p+q_{s}}\right)^{k_{s}}\right)^{n-k_{s}+1} .
\end{aligned}
$$

Tables 1 and 2 display the values of all aforementioned bounds for a variety of choices of $m, n$ and $k_{s}, q_{s}, s=1,2, \ldots, m$. It is clear that for highly reliable systems the group of much simpler bounds $L_{\text {Bonf }}^{\prime}, U^{\prime}, L^{\prime}, L_{S F M}^{\prime}$ (as compared to $L_{B o n f}, U, L, L_{S F M}$ ) is still offering quite good estimates for the unknown system reliability.

As far as the asymptotic behaviour of the system is concerned, observe that for fixed $m, k_{1} k_{2}, \ldots, k_{m}$ as $\nu=n \rightarrow \infty$, the quantities $c_{s}(n), l_{s}(n)$ are bounded $\left(c_{s}(n)=k_{s}, L_{s 1}=\emptyset, L_{s j}=\{j-1\}, j \geq 2\right)$; moreover, if $\lim _{n \rightarrow \infty}\left(n-k_{s}+1\right) q_{s}^{k_{s}}(n) \rightarrow \lambda_{s}<\infty$ for $s=1,2, \ldots, m$ then $\lim _{n \rightarrow \infty} R_{M F M}(n)=$ $e^{-\left(\lambda_{1}+\ldots+\lambda_{m}\right)}$. Thus, loosely speaking, if the components' reliability $p=1-\sum_{i=1}^{m} q_{i}$ is close to 1 and the number $n$ of system's components is large then

$$
R_{M F M} \approx e^{-n\left(q_{1}^{k_{1}}+\ldots+q_{m}^{k_{m}}\right)}
$$

This approximation is clearly illustrated in Table 3 where we have computed several reliability bounds for consecutive-4,3,2-out-of- $n$ : $M F M$ systems with components' failure probabilities $q_{s}=\left(\lambda_{s} / n\right)^{1 / k_{s}}, \lambda_{1}=\lambda_{2}=\lambda_{3}=0.15$ (so that the asymptotic reliability of Theorem 5 becomes $\left.R_{M F M} \approx e^{-0.15-0.15-0.15} \approx 0.6376\right)$. Note that the Bonferroni-type bound ( $\left.L_{\text {Bonf }}^{\prime}\right)$ is not adequate for attaining the asymptotic reliability value. 


\begin{tabular}{cccccccccccc}
\hline$p$ & $q_{1}$ & $q_{2}$ & $q_{3}$ & $L_{S F M}^{\prime}$ & $L^{\prime}$ & $L_{\text {Bonf }}^{\prime}$ & $L_{\text {Bonf }}$ & $L$ & $L_{S F M}$ & $U^{\prime}$ & $U$ \\
\hline 0.5 & 0.25 & 0.1667 & 0.0833 & 0.0020 & 0.0856 & -0.597 & -0.405 & 0.1360 & 0.0002 & 0.1494 & 0.1486 \\
0.55 & 0.225 & 0.15 & 0.075 & 0.0027 & 0.2177 & -0.177 & -0.032 & 0.2766 & 0.0094 & 0.2825 & 0.2819 \\
0.6 & 0.2 & 0.1333 & 0.0667 & 0.0468 & 0.3927 & 0.2007 & 0.2939 & 0.4450 & 0.0797 & 0.4474 & 0.4470 \\
0.65 & 0.175 & 0.1167 & 0.0583 & 0.2187 & 0.5784 & 0.5008 & 0.5528 & 0.6150 & 0.2713 & 0.6159 & 0.6157 \\
0.7 & 0.15 & 0.1 & 0.05 & 0.4918 & 0.7405 & 0.7150 & 0.7400 & 0.7611 & 0.5326 & 0.7614 & 0.7613 \\
0.75 & 0.125 & 0.0833 & 0.0416 & 0.7375 & 0.8601 & 0.8535 & 0.8638 & 0.8694 & 0.7569 & 0.8695 & 0.8694 \\
0.8 & 0.1 & 0.0667 & 0.0333 & 0.8908 & 0.9355 & 0.9343 & 0.9378 & 0.9389 & 0.8971 & 0.9389 & 0.9389 \\
0.85 & 0.075 & 0.05 & 0.025 & 0.9644 & 0.9761 & 0.976 & 0.9768 & 0.9770 & 0.9658 & 0.9770 & 0.9770 \\
0.9 & 0.05 & 0.0333 & 0.0166 & 0.992 & 0.9939 & 0.9938 & 0.9940 & 0.9940 & 0.9922 & 0.9940 & 0.9940 \\
\hline
\end{tabular}

Table 2: Reliability bounds for consecutive-5,4,3-out-of-1000 : $M F M$ systems

\begin{tabular}{cccccccc}
\hline$n$ & $q_{1}$ & $q_{2}$ & $q_{3}$ & $L_{S F M}^{\prime}$ & $L^{\prime}$ & $L_{\text {Bonf }}^{\prime}$ & $U^{\prime}$ \\
\hline 10 & 0.35 & 0.2466 & 0.1225 & 0.0881 & 0.6713 & 0.6585 & 0.7485 \\
100 & 0.1968 & 0.1145 & 0.03873 & 0.3959 & 0.6399 & 0.5896 & 0.6767 \\
1000 & 0.1107 & 0.05313 & 0.01225 & 0.5314 & 0.6378 & 0.5829 & 0.6552 \\
10000 & 0.06223 & 0.02466 & 0.003873 & 0.5872 & 0.6376 & 0.5822 & 0.6464 \\
100000 & 0.035 & 0.01145 & 0.001225 & 0.6124 & 0.6376 & 0.5821 & 0.6422 \\
1000000 & 0.01968 & 0.005313 & 0.0003873 & 0.6246 & 0.6376 & 0.5821 & 0.6401 \\
10000000 & 0.01107 & 0.002466 & 0.0001225 & 0.6307 & 0.6376 & 0.5821 & 0.6389 \\
\hline
\end{tabular}

Table 3: Approximating the asymptotic reliability of a consecutive-4, 3, 2-out-of- $n: M F M$ system

b) $k_{1}, k_{2}, \ldots, k_{m}$-out-of- $n$ :MFM system

A $k_{1}, k_{2}, \ldots, k_{m}$-out-of- $n: M F M$ system consists of $n$ components and enters failure mode $s$ when at least $k_{s}$ components fail in mode $s, s=1,2, \ldots, m$. Its minimal cut sets of type $s$ are given by $\mathbf{C}_{s}=\left\{C \subset I:|C|=k_{s}\right\}, s=1,2, \ldots, m$. Denoting by $R_{s}(q)=\sum_{i=0}^{k_{s}-1}\left(\begin{array}{c}n \\ i\end{array}\right) q^{i}(1-q)^{n-i}$ the reliability of a $k_{s}$-out-of- $n: S F M$ system with component failure probabilities $q$ (for a review see Rushdi [22]) we may easily obtain the following reliability bounds (cf. (18), (23) and (26))

$$
\begin{aligned}
& L_{\text {Bonf }}=\sum_{s=1}^{m} R_{s}\left(q_{s}\right)-m+1, \quad U=\prod_{s=1}^{m} R_{s}\left(q_{s}\right), \quad L=\prod_{s=1}^{m} R_{s}\left(q_{s}\right)-\sum_{s<t} c_{s t}, \\
& L_{S F M}=\prod_{s=1}^{m} R_{s}\left(Q_{s}\right), \quad Q_{s}=\frac{q_{s}}{q_{s}+p}, s=1,2, \ldots, m
\end{aligned}
$$

where $c_{s t}=q_{s}^{k_{s}} q_{t}^{k_{t}}\left(\begin{array}{c}n \\ k_{s}\end{array}\right)\left(\left(\begin{array}{c}n \\ k_{t}\end{array}\right)-\left(\begin{array}{c}n-k_{s} \\ k_{t}\end{array}\right)\right)$. Moreover, the lower bound (24) takes on the form

$$
\left.L^{*}=\prod_{s=1}^{m} R_{s}\left(q_{s}\right)-\sum_{s<t}\left(R_{s}\left(q_{s}\right) R_{t}\left(q_{t}\right)-\sum_{i=0}^{k_{s}-1} \sum_{j=0}^{k_{t}-1}\left(\begin{array}{c}
n \\
i, j
\end{array}\right) q_{s}^{i} q_{t}^{j}\left(1-q_{s}-q_{t}\right)^{n-i-j}\right)\right) .
$$

In Table 4 we conduct a numeric comparison of these bounds for several values of $n, k_{i}, q_{i}$ and $m$. Exploiting the aforementioned bounds we can easily investigate the asymptotic behaviour of 


\begin{tabular}{cccccc}
\hline$q_{s}$ & $L_{S F M}$ & $L^{*}$ & $L$ & $L_{\text {Bonf }}$ & $U$ \\
\hline 0.02 & 0.0066 & 0.2942 & -2.189 & -0.016 & 0.3523 \\
0.0175 & 0.0724 & 0.5019 & -0.142 & 0.3688 & 0.5266 \\
0.015 & 0.2906 & 0.6912 & 0.5567 & 0.6461 & 0.6997 \\
0.0125 & 0.6054 & 0.8387 & 0.8178 & 0.8275 & 0.8409 \\
0.01 & 0.8481 & 0.9332 & 0.9311 & 0.9314 & 0.9335 \\
0.0075 & 0.9620 & 0.9804 & 0.9802 & 0.9802 & 0.9804 \\
0.005 & 0.9950 & 0.9968 & 0.9968 & 0.9968 & 0.9968 \\
\hline
\end{tabular}

Table 4: Reliability bounds for 5,5,..,5-out-of-100 : $M F M$ systems with $m=20$

\begin{tabular}{ccccccc}
\hline$n$ & $q_{s}$ & $L_{S F M}$ & $L^{*}$ & $L$ & $L_{\text {Bonf }}$ & $U$ \\
\hline 100 & 0.01 & 0.3551 & 0.4224 & 0.3336 & 0.2063 & 0.4374 \\
200 & 0.005 & 0.3951 & 0.4276 & 0.3811 & 0.2016 & 0.4351 \\
300 & 0.003333 & 0.4079 & 0.4294 & 0.3979 & 0.2001 & 0.4344 \\
400 & 0.0025 & 0.4143 & 0.4303 & 0.4065 & 0.1993 & 0.4341 \\
500 & 0.002 & 0.4181 & 0.4308 & 0.4117 & 0.1988 & 0.4338 \\
600 & 0.001667 & 0.4206 & 0.4312 & 0.4152 & 0.1985 & 0.4337 \\
700 & 0.001429 & 0.4224 & 0.4314 & 0.4177 & 0.1983 & 0.4336 \\
800 & 0.00125 & 0.4237 & 0.4316 & 0.4196 & 0.1981 & 0.4335 \\
900 & 0.001111 & 0.4247 & 0.4318 & 0.4211 & 0.198 & 0.4334 \\
1000 & 0.001 & 0.4256 & 0.4319 & 0.4222 & 0.1979 & 0.4334 \\
\hline
\end{tabular}

Table 5: Approximating the asymptotic reliability of a 3,.., 3-out-of- $n: M F M$ system with $m=10$ 
the system. Let us assume that $m, k_{s}$ are fixed as $\nu=n \rightarrow \infty$ and $\lim _{n \rightarrow \infty} n q_{s}(n)=\lambda_{s}$. Then,

$$
\lim _{n \rightarrow \infty} c_{s t}(n)=0, \quad \lim _{n \rightarrow \infty} R_{s}\left(q_{s}\right)=\lim _{n \rightarrow \infty} \sum_{i=0}^{k_{s}-1}\left(\begin{array}{c}
n \\
i
\end{array}\right) q_{s}^{i}\left(1-q_{s}\right)^{n-i}=\sum_{i=0}^{k_{s}-1} e^{-\lambda_{s}} \frac{\lambda_{s}^{i}}{i !}
$$

and the asymptotic reliability of the $M F M$ system will be given by

$$
\lim _{n \rightarrow \infty} R_{M F M}=\prod_{s=1}^{m} \sum_{i=0}^{k_{s}-1} e^{-\lambda_{s}} \frac{\lambda_{s}^{i}}{i !}
$$

The above result is clearly illustrated in Table 5 which displays the reliability bounds for a $3,3, \ldots, 3$-out-of- $n$ : MFM system with $m=10$ failure modes and components' failure probabilities $q_{s}=\lambda_{s} / n, \lambda_{s}=1, s=1,2, \ldots, m$. Note that, with the exception of the Bonferroni bound, all other bounds approach very rapidly (as $n$ increases) the asymptotic reliability value $R_{M F M} \approx \prod_{s=1}^{10} \sum_{i=0}^{2} e^{-\lambda_{s}} \frac{\lambda_{s}^{i}}{i !}=\left(\frac{5}{2} e^{-1}\right)^{10} \approx 0.4330$.

\section{References}

[1] Aki, S. (1992). Waiting time problems for a sequence of discrete random variables. Annals of the Institute of Statistical Mathematics 44, 363-378.

[2] Barlow, R. and Proschan, F. (1975). Statistical Theory of Reliability and Life Testing, Holt Reinhart and Winston, Inc.

[3] Barlow, R. and Wu, A. (1978). Cohereht systems with multi-state components. Mathematics of Operations Research 3, 275-281.

[4] Barlow, R., Hunter, L. and Proschan, F. (1963). Optimum redundancy when components are subject to two kinds of failure. J. Soc. Ind. Appl. Math. 11, 64-73.

[5] Ben-Dov, Y. (1980). Optimal reliability design of $k$-out-of- $n$ systems subject to two kinds of failure. Journal of Operational Research Society 31, 743-748.

[6] Boutsikas, M.V. and Koutras, M.V. (2000a). A bound for the distribution of the sum of discrete associated or NA random variables. Annals of Applied Probability 10, 1137-1150.

[7] Boutsikas, M.V. and Koutras, M.V. (2000b). Generalized reliability bounds for coherent structures. Journal of Applied Probability 37, 778-794.

[8] Chao, M. T., Fu, J. C. and Koutras M. V. (1995). Survey of reliability studies of consecutive $k$-out-of- $n: F$ \& related systems. IEEE Transactions on Reliability 44, 120-127.

[9] Chryssaphinou, O. and Vaggelatou, E. (2001). Compound Poisson approximation for multiple runs in a Markov chain. Annals of the Institute of Statistical Mathematics (to appear)

[10] El-Neweihi, E., Proschan, F. and Sethuraman, J. (1978). Multistate coherent systems. Journal of Applied Probability 15, 675-688. 
[11] Esary, J.D. and Proschan, F. (1963). Coherent structures of non-identical components. Technometrics 5, 191-209.

[12] Esary, J.D., Proschan, F. and Walkup, D. (1967). Association of random variables with applications. The Annals of Mathematical Statistics 38, 1466-1474.

[13] Fu, J. C. and Koutras, M. V. (1995) Reliability bounds for coherent structures with independent components. Statistics and Probability Letters 22, 137-148.

[14] Griffith, W. S. (1980). Multistate reliability models. Journal of Applied Probability 17, 735-744.

[15] Joag-Dev, K. and Proschan, F. (1983). Negative association of random variables with applications. The Annals of Statistics 11, 286-295.

[16] Koutras, M.V. (1997). Consecutive $k, r$-out-of-n : DFM systems. Microelecronics Reliability 37, 597-603.

[17] Koutras, M.V. and Alexandrou V. A. (1996). Sooner waiting time problems in a sequence of trinomial trials. Journal of Applied Probability 34, 593-609.

[18] Meng, F. C. (1994). Characterizing the Barlow-Wu structure functions. Naval Research Logistics 41, 661-668.

[19] Misra, K.B. (1992). Reliability Analysis and Prediction: A methodology oriented treatment, Elsevier.

[20] Natvig, B. (1982). Two suggestions of how to define a multistate cohereht system. Annals of Applied Probability 14, 434-455.

[21] Ross, S. M. (1979). Multivalued state component systems. The Annals of Probability 7, 379383.

[22] Rushdi, A.M. (1993). Reliability of $k$-out-of- $n$ systems. In New Trends in System Reliability Evaluation (K.B. Misra Ed.), pp 185-227. Elsevier Science Publ. B.V.

[23] Satoh, N., Sasaki, M., Yuge, T. and Yanasi, S. (1993). Reliability of 3-state device systems with simultaneous failures. IEEE Transactions on Reliability 42, 470-477. 